\title{
Surfactant protein D, a clinical biomarker for chronic obstructive pulmonary disease with excellent discriminant values
}

\author{
ZEINA AKIKI ${ }^{1-5}$, DALIA FAKIH ${ }^{4,6,7}$, RANIA JOUNBLAT ${ }^{4,7}$, SOULAIMA CHAMAT $^{4,8}$, MIRNA WAKED $^{9,10}$, \\ UFFE HOLMSKOV $^{6}$, GRITH L. SORENSEN ${ }^{6}$, RACHEL NADIF $^{1-2}$ and PASCALE SALAMEH ${ }^{4,5}$
}

${ }^{1}$ INSERM, VIMA: Aging and Chronic Diseases, Epidemiological and Public Health Approaches, U1168, F-94807 Villejuif;

${ }^{2}$ University of Versailles Saint-Quentin-en-Yvelines, F-94807 Villejuif; ${ }^{3}$ University of Paris-Sud, F-94270 Paris, France;

${ }^{4}$ Laboratory of Immunology, Faculty of Public Health, Doctoral School for Sciences and Technology, Lebanese University,

90656 Jdeidet El Metn; ${ }^{5}$ Clinical and Epidemiological Research Laboratory, Faculty of Pharmacy, Lebanese University,

Beirut 6573-14, Lebanon; ${ }^{6}$ Department of Cancer and Inflammation, Institute of Molecular Medicine, University of Southern

Denmark, DK-5000 Odense C, Denmark; ${ }^{7}$ Laboratory of Histology, Cellular and Molecular Biology and Immunology,

Faculty of Sciences II, Lebanese University, 90656 Jdeidet El Metn; ${ }^{8}$ Faculty of Medicine, Lebanese University,

Beirut 6573-14; ${ }^{9}$ Saint George Hospital University Medical Center, Beirut 1100-2807;

${ }^{10}$ Faculty of Medicine, Balamand University, Beirut 1100-2807, Lebanon

Received April 4, 2015; Accepted November 3, 2015

DOI: 10.3892/etm.2016.2986

\begin{abstract}
Biological markers can help to better identify a disease or refine its diagnosis. In the present study, the association between surfactant protein D (SP-D) and chronic obstructive pulmonary disease (COPD) was studied among subjects consulting for respiratory diseases or symptoms and was compared with C-reactive protein (CRP) and fibrinogen. A further aim of this study was to identify the optimal cut-off point of SP-D able to discriminate COPD patients. A case-control study including 90 COPD patients, 124 asthma patients and 180 controls was conducted. Standardized questionnaires were administered and lung function tests were performed. Biological markers were measured in blood samples according to standardized procedures. The association between SP-D and COPD was investigated using logistic regression models. Receiver-operating characteristic curves were used for threshold identification. SP-D levels above the median value were positively associated with COPD [adjusted odds ratio $(\mathrm{OR})=3.86,95 \%$ confidence interval $(\mathrm{CI}): 1.51-9.85$, $\mathrm{P}=0.005)$. No associations with COPD or asthma were found for CRP or fibrinogen levels. Scores for COPD diagnosis in all COPD patients or ever-smoker COPD patients were identified (sensitivity, 76.4 and $77.8 \%$; specificity, 89.3 and $88.5 \%$, respectively). The results indicate that SP-D can differentiate
\end{abstract}

Correspondence to: Mrs. Zeina Akiki, Clinical and Epidemiological Research Laboratory, Faculty of Pharmacy, Lebanese University, 37 Hadath, Beirut 6573-14, Lebanon

E-mail: zeina.akiki@inserm.fr; zeinaakiki@hotmail.com

Key words: serum surfactant protein D, serum C-reactive protein, plasma fibrinogen, chronic obstructive pulmonary disease, asthma
COPD from other respiratory symptoms or diseases. Used with socio-demographic characteristics and respiratory symptoms, SP-D is able to discriminate COPD patients from controls, particularly among smokers.

\section{Introduction}

Chronic obstructive pulmonary disease (COPD) is ranked as the fourth leading cause of mortality worldwide (1). A diagnosis of COPD is considered in any patient who has dyspnea, chronic cough or sputum production and a history of exposure to risk factors, but requires confirmation by spirometry (1). This technique is, however, difficult for the patient, not always available, requires a certain expertise for its interpretation and is poorly correlated with disease severity $(2,3)$. As an alternative, research has focused recently on the measurement of a variety of biomarkers that appear to exhibit prospective utility in the diagnosis and prognosis of COPD. Serum levels of systemic markers of inflammation such as C-reactive protein (CRP) and fibrinogen, which are validated in clinical laboratory assays (4), have been shown to reflect the degree of severity of airway inflammation (5-7) but they are not lung-specific (7-10). In addition, there is no approved predictive or prognostic biomarker for COPD reported to date.

Previous studies have described associations between a pulmonary-specific marker, surfactant protein D (SP-D) and COPD $(11,12)$. SP-D is a large hydrophilic glycoprotein that belongs to the collectin family, mainly produced in the lung by alveolar type II cells and non-ciliated Clara cells (13). SP-D facilitates the resolution of lung inflammation (14), is detectable in serum, and has the advantage of being stable over a period of 6 months (15).

In the present study, it was hypothesized that SP-D is a more specific biological marker than CRP or fibrinogen for the differentiation of COPD patients among individuals 
consulting for respiratory diseases or symptoms, including those with asthma. Therefore, the associations of SP-D, CRP and fibrinogen with patients with COPD or asthma and controls were investigated. The optimal cut-off point able to discriminate COPD patients from controls using serum SP-D levels was also sought.

\section{Materials and methods}

Study design and subjects. All COPD patients consulting at the outpatient clinics of the Pulmonary Department of Saint George Hospital University Medical Center in Beirut, Lebanon between June 2011 and April 2013 were recruited in this case-control study. COPD diagnosis was defined as a post bronchodilator ratio of the forced expiratory volume in $1 \mathrm{sec}\left(\mathrm{FEV}_{1}\right)$ /forced vital capacity (FVC) of $<70 \%$ (1). Ninety COPD patients were classified into categories based on Global Initiative for Chronic Obstructive Pulmonary Disease (GOLD) guidelines 2013 (1). There were 35 patients in group A, 38 in group B, 3 in group C, and 14 in group D. None of the patients had an exacerbation 1 month prior to inclusion. With regard to medication, COPD patients were classified as treated when they were receiving $\beta 2$-agonists or anti-cholinergic agents (whether short-acting or long-acting) combined or not with inhaled corticosteroids (ICS), as suggested previously (16). A total of 46 COPD patients were found to be on regular medication. Patients with asthma $(n=124)$ were defined according to Global Initiative for Asthma (GINA) guidelines 2012 (17). A clinical diagnosis based on symptoms (breathlessness, wheezing, cough and chest tightness), family history of asthma, worsening symptoms when exposed to various risk factors was performed and lung function measurements were assessed (17). Having another respiratory disease was the exclusion criterion for COPD or asthma patients. Healthy individuals from the general population $(n=92)$ and outpatients consulting for a variety of non-respiratory problems in the same hospital $(\mathrm{n}=88)$ were recruited. Controls had normal lung function test results as defined by GOLD guidelines (1). The exclusion criteria were previous or current diagnosis of any respiratory disease such as asthma, COPD, chronic bronchitis, fibrosis, tuberculosis or lung cancer. Written informed consent was provided by all participants.

Questionnaire. A standardized questionnaire, adapted to the local Arabic language, was filled out by all participants with the assistance of trained interviewers. The questionnaire included information on socio-demographic characteristics, exposure to disease risk factors, clinical assessment of the disease and its symptoms using the American Thoracic Society Questionnaire (18) and the Medical Research Council 'MRC' breathlessness scale (19), and smoking history.

Blood collection and pulmonary function tests. Before conducting pulmonary function tests, venous blood samples were withdrawn from all participants, immediately transported at $4^{\circ} \mathrm{C}$ to the Immunology Laboratory of the Lebanese University (Fanar, Lebanon) and centrifuged at $4^{\circ} \mathrm{C}$ within $12 \mathrm{~h}$ after withdrawal. Plasma and serum were then aliquoted and stored frozen at $-20^{\circ} \mathrm{C}$ until their analysis within 6 months.
All participants underwent baseline spirometry by a trained physician. Reversibility assessments (post-bronchodilator spirometry) were performed following the inhalation of two puffs of Ventolin (albuterol) with 30 min delay from the baseline spirometry.

Measurement of SP-D, CRP and fibrinogen. Quantification of SP-D was performed by a five-layered enzyme-linked immunosorbent assay (ELISA) as previously described by Leth-Larsen et al (20). Briefly, microtiter plates were coated with polyclonal $\left.\mathrm{F}(\mathrm{ab})_{2}\right)_{2}$ anti-human SP-D antibody [cat.no., K477; dilution, 1:1500; Grith Sorensen's laboratory, Odense, Denmark (20)] in sodium carbonate buffer (pH 9.6). Following overnight incubation at $4^{\circ} \mathrm{C}$, the plates were washed and left in contact with washing buffer (Tris-buffered saline, $0.05 \%$ Tween $20,5 \mathrm{mMCaCl}_{2}$ ) for $1 \mathrm{~h}$ at room temperature. Calibrator, controls and samples were added at a dilution of 1:10 and incubated overnight at $4^{\circ} \mathrm{C}$. This was followed by successive incubations with biotinylated monoclonal anti-human SP-D antibody [dilution, 1:2,000; Grith Sorensen's laboratory (20)], horseradish peroxidase-conjugated streptavidin (cat.no., 43-4323; dilution, 1:20,000; Zymed; Thermo Fisher Scientific, Inc., Waltham, MA, USA) and $o$-phenylenediamine (Zymed; Thermo Fisher Scientific, Inc.) in citrate-phosphate buffer $\mathrm{pH} 5$, containing $0.014 \% \mathrm{H}_{2} \mathrm{O}_{2}$. Plates were read at $492 \mathrm{~nm}$ in a multichannel spectrophotometer (Bio-Tek ELx800 (BioTek instruments, Inc., Vermont, USA) after adding $\mathrm{H}_{2} \mathrm{SO}_{4}$. Serum $\mathrm{CRP}$ and plasma fibrinogen levels were measured using double antibody sandwich ELISA kits (Immunology Consultants Laboratory, Inc., Portland, OR, USA) according to the manufacturer's protocol. All samples were tested in duplicate. The coefficient of variation was $4.8 \%$ for SP-D, $2.7 \%$ for CRP, and $2 \%$ for fibrinogen.

Statistical analysis. Fibrinogen was normally distributed, whereas SP-D and CRP levels were not, even following log transformation. Results are expressed as the median (interquartile range). Differences between groups were tested using the t-test or Chi-square test when appropriate (non-parametric tests gave the same results). Associations between biomarkers and lung function tests were estimated using general linear models with adjustment for age, gender, body mass index (BMI) classes and smoking status. Three logistic regression models were used to evaluate the association between SP-D and COPD. In the first and second regressions, COPD patients vs. controls were used as the dependent variable. The independent variables were SP-D expressed as above/below the median value, and all potential confounding variables such as age, gender, BMI class, and smoking, and all the remaining socio-demographic characteristics and respiratory symptoms (cough, wheezing and expectoration) having $\mathrm{P}<0.2$ in the bivariate analysis. As a sensitivity analysis, the second regression was performed in ever smokers (smokers and ex-smokers) only. Then, in order to confirm the ability of SP-D to differentiate patients with COPD from patients with asthma, a third regression including COPD vs. asthma patients as the dependent variable was performed. The adjusted odds ratios (aORs) obtained from the first and second regressions respectively were then rounded to the nearest unit and used as coefficients in the calculation of score 1 (for all COPD patients 
Table I. Characteristics of controls, COPD patients and asthma patients.

\begin{tabular}{lccc}
\hline Characteristic & Controls $(\mathrm{n}=180)$ & COPD patients $(\mathrm{n}=90)$ & Asthma patients $(\mathrm{n}=124)$ \\
\hline Age, years & $55(51-64)$ & $62(50-71)$ & $46(32-61)$ \\
BMI classes $^{\mathrm{a}}$ & & & \\
$\quad$ Normal & $52(29.9)$ & $33(37.1)$ & $36(37.4)$ \\
Overweight & $82(47.1)$ & $23(25.8)$ & $44(35.8)$ \\
Obese & $40(23.0)$ & $52(57.8)$ & $33(26.8)$ \\
Male gender & $65(36.5)$ & $61(71.8)$ & $39(32.2)$ \\
Married & $134(84.3)$ & $12(13.8)$ & $70(60.9)$ \\
University education & $28(16.4)$ & $35(41.7)$ & $43(35.0)$ \\
In work & $69(39.9)$ & $68(75.6)$ & $55(45.8)$ \\
Ever smoker & $112(62.2)$ & $60(48.4)$
\end{tabular}

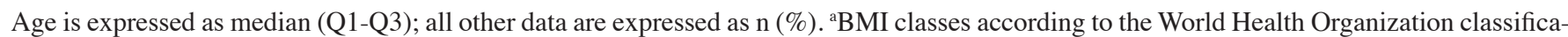
tion. COPD, chronic obstructive pulmonary disease; BMI, body mass index; Q, quartile.

Table II. Variations of SP-D, CRP and fibrinogen levels between patients with COPD or asthma and controls.

\begin{tabular}{lccccc}
\hline & & & & P-value \\
\cline { 4 - 6 } & Controls & COPD & Asthma \\
Analyte & $(\mathrm{n}=180)$ & $\begin{array}{c}\text { COPD vs. } \\
(\mathrm{n}=124)\end{array}$ & $\begin{array}{c}\text { Asthma vs. } \\
\text { controls }\end{array}$ & $\begin{array}{c}\text { COPD vs. } \\
\text { controls } \\
\text { asthma }\end{array}$ \\
\hline SP-D $(\mathrm{ng} / \mathrm{ml})$ & $1,269(664-1884)$ & $1,510(986-2,174)$ & $1,130(676-1,852)$ & 0.02 & 0.7 \\
CRP $(\mathrm{ng} / \mathrm{ml})$ & $9.72(4.37-15.5)$ & $8.41(3.48-14.3)$ & $8.35(3.35-15.8)$ & 0.5 & 0.4 \\
Fibrinogen $(\mu \mathrm{g} / \mathrm{ml})$ & $3,135(2,730-3,597)$ & $2,992(2,550-3,965)$ & $3,358(2,575-4,079)$ & 0.5 & 0.09 \\
\hline
\end{tabular}

Results are expressed as median (Q1-Q3). COPD, chronic obstructive pulmonary disease; SP-D, surfactant protein-D; CRP, C-reactive protein; $\mathrm{Q}$, quartile.

and controls) and score 2 (for ever-smoker COPD patients and controls) with the purpose of predicting COPD diagnosis. Receiver-operating characteristic (ROC) curves were then generated in order to determine the ability of the scores 1 and 2 to discriminate between patients with COPD and controls. $\mathrm{P}<0.05$ was considered to indicate a statistically significant difference. All analyses were performed using SPSS software, version 17.0 (SPSS, Inc., Chicago, IL, USA).

According to a previous study on SP-D in COPD (21), the smallest difference that could exist between healthy individuals and COPD patients was $56.6 \mathrm{ng} / \mathrm{ml}$, while the standard deviation was $80.2 \mathrm{ng} / \mathrm{ml}$. With an $\alpha$ error of $5 \%$ and a power of $80 \%$, a minimum of 32 patients and 46 controls was required for the study.

\section{Results}

Participants' characteristics. Characteristics of the COPD and asthma patients and controls are presented in Table I. The three groups were significantly different regarding age, gender, smoking, marital status and education. Compared with controls, patients with COPD were more frequently men, ever smokers and unmarried (all $\mathrm{P} \leq 0.003$ ); while patients with asthma were younger, more often unmarried, non-smokers and were more likely to have a university degree (all $\mathrm{P} \leq 0.02$ ). Compared with asthma patients, COPD patients were older, more frequently men, ever smokers and were less likely to have a university degree (all $\mathrm{P} \leq 0.001$ ). No other significant associations were found.

Association of biological markers with COPD and asthma. The three groups were significantly different regarding SP-D levels only. Serum SP-D levels were significantly increased in COPD patients as compared with controls and with asthma patients. There were no significant differences in SP-D levels between asthma patients and controls. No other significant associations were observed (Table II).

SP-D levels were lower in the COPD patients that were receiving inhaled therapy (ICS and/or bronchodilators) compared with the COPD patients that were not, but the difference was not statistically significant [n=46 vs. $42 ; 1,507 \pm 868$ vs. $1,830 \pm 971 \mathrm{ng} / \mathrm{ml}$, respectively, $\mathrm{P}=0.1]$.

No significant associations were identified between biological markers and FEV1\% predicted or FEV1/FVC following the administration of a bronchodilator to the COPD patients (data not shown). 
Table III. Multivariate analysis of the association between serum SP-D levels and COPD.

A, First regression: COPD patients vs. controls as the dependent variable $(n=221)$

\begin{tabular}{lccc}
\hline Characteristic & aOR & 95\% CI & P-value \\
\hline SP-D above the median & 3.86 & $1.51 ; 9.85$ & 0.005 \\
Male gender & 2.92 & $1.23 ; 6.93$ & 0.02 \\
Unmarried & 3.07 & $1.19 ; 7.96$ & 0.02 \\
Cough in the morning & 39.2 & $10.1 ; 153$ & $<0.001$ \\
Cough during the day & 10.9 & $3.25 ; 36.6$ & $<0.001$ \\
Wheezing during the day & 64.9 & $11.8 ; 356$ & $<0.001$ \\
Ever smoker & 1.31 & $0.54 ; 3.18$ & 0.5 \\
\hline
\end{tabular}

B, Second regression: COPD patients vs. controls as the dependent variable for ever smokers $(n=141)$

\begin{tabular}{lccc}
\hline Characteristic & aOR & $95 \%$ CI & P-value \\
\hline SP-D above the median & 6.26 & $1.81 ; 21.65$ & 0.004 \\
Male gender & 4.22 & $1.43 ; 12.5$ & 0.009 \\
Unmarried & 3.38 & $1.04 ; 10.9$ & 0.04 \\
Cough in the morning & 53.8 & $10.7 ; 272$ & $<0.001$ \\
Cough during the day & 8.66 & $1.80 ; 41.8$ & 0.007 \\
Wheezing during the day & 35.8 & $4.82 ; 267$ & $<0.001$ \\
\hline
\end{tabular}

C, Third regression: COPD patients vs. asthma patients as the dependent variable $(n=201)$

\begin{tabular}{lccr}
\hline Characteristic & aOR & $95 \%$ CI & P-value \\
\hline SP-D above the median & 2.53 & $1.29 ; 4.96$ & 0.007 \\
Male gender & 2.84 & $1.44 ; 5.62$ & 0.003 \\
Age & 1.04 & $1.02 ; 1.07$ & $<0.001$ \\
Cough in the morning & 8.70 & $3.29 ; 23.0$ & $<0.001$ \\
\hline
\end{tabular}

For A, Nagelkereke's $\mathrm{R}^{2}=60 \%$; Hosmer-Lemeshow goodness-of-fit, $\mathrm{P}=0.541 ; 86.4 \%$ of the subjects were correctly classified. For $\mathrm{B}$, Nagelkereke's $R^{2}=62 \%$; Hosmer \& Lemeshow $=0.926 ; 84.4 \%$ of the subjects were correctly classified. For C, Nagelkereke's $\mathrm{R}^{2}=39 \%$; Hosmer $\&$ Lemeshow $=0.867 ; 76.1 \%$ of the subjects were correctly classified. aOR, adjusted odds ratio; CI, confidence interval; SP-D, surfactant protein-D; COPD, chronic obstructive pulmonary disease.

Multivariate analyses of the association between SP-D and $C O P D$. Multivariate analyses performed on all COPD patients and controls showed that SP-D levels above the median value, male gender, being unmarried and symptoms such as having a morning cough, cough during the day and wheezing during the day were significantly and positively associated with COPD (Table IIIA). The same associations were observed in ever-smoker COPD patients and controls (Table IIIB).

In the third regression (Table IIIC) with COPD patients vs. asthma patients as the dependent variable, SP-D levels above the median value, older age, male gender and having a morning cough were significantly and positively associated with COPD.

Construction, properties and thresholds of scores for COPD diagnosis. Taking into account the aORs from the first regression (from Table IIIA) and rounding to the nearest unit, a first score (score 1) for COPD diagnosis was computed as follows: Score $1=($ SP-D above/below the median $x 4)+($ gender $x 3)$
$+($ marital status x 3$)+($ cough in the morning $x 39)+($ cough during the day $x$ 11) + (wheezing during the day $\times 65)+$ (smoking x 1).

In COPD patients, score 1 had a minimum of 6 and a maximum of 129 . The mean was 47.2 , the median was 49 and the standard deviation was 31.9. In controls, the minimum was 6 and the maximum was 75, with a mean of 12.6, a median of 10 and a standard deviation of 10.1 .

The ROC curve generated for score 1 is shown in Fig. 1, for the comparison of COPD patients with controls. The area under the curve was $0.890(0.841-0.940 ; \mathrm{P}<0.001)$. The most optimal cut-off point was calculated to be 15.5 (Table IV) at which point the sensitivity, specificity, positive predictive value and negative predictive value were $76.4,89.3,81$ and $74 \%$, respectively.

A second score (score 2) was calculated from the second regression (from Table IIIB) for ever-smoker COPD patients and controls as follows: Score $2=$ (SP-D above/below the 


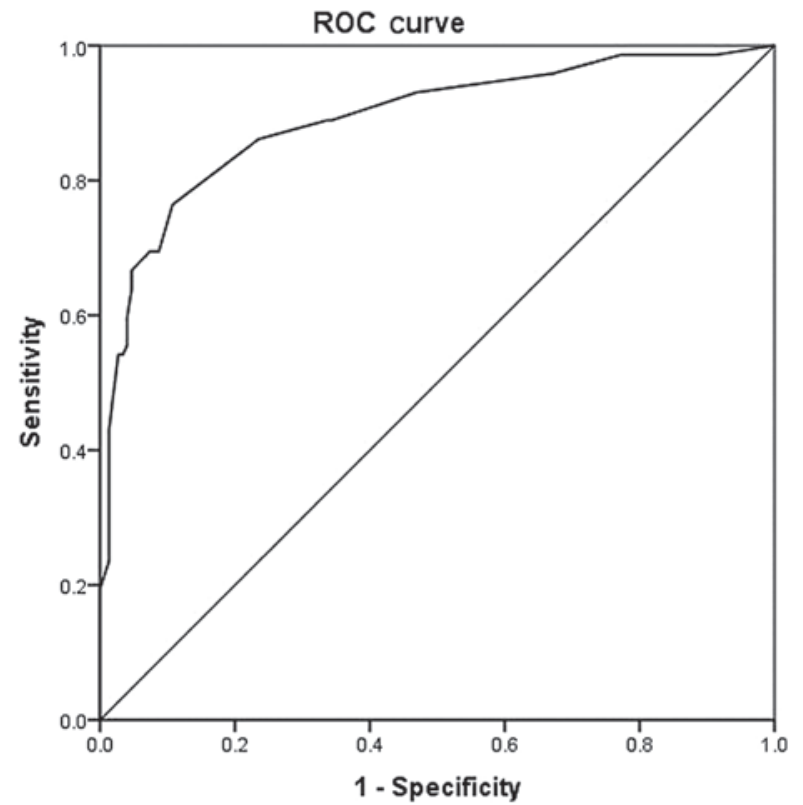

Figure 1. Receiver-operating characteristic (ROC) curve for all patients with chronic obstructive pulmonary disease and controls. Diagonal segments are produced by ties.

median $\times 6)+($ gender $\times 4)+($ marital status $\times 3)+($ cough in the morning $x$ 54) $+($ cough during the day $x 9)+($ wheezing during the day $\mathrm{x} 36$ ).

In ever-smoker COPD patients, score 2 had a minimum of 10 and a maximum of 116 . The mean was 47.7 , the median was 51 and the standard deviation of 28.5. In ever-smoker controls, the minimum was 7 and the maximum was 67, with a mean of 15 , a median of 13 and a standard deviation of 11.4.

A ROC curve was generated from score 2 (Fig. 2). The area under the curve was at $0.895(0.841-0.950 ; \mathrm{P}<0.001)$. The most optimal cut-off point was 18.5 at which point the sensitivity, specificity, positive predictive value and negative predictive value were $77.8,88.5,70$ and $82 \%$ respectively (Table V).

\section{Discussion}

In this study, the associations between serum SP-D and COPD among subjects consulting for respiratory diseases or symptoms, and in comparison with serum CRP and plasma fibrinogen levels were investigated. SP-D levels were found to be significantly and positively associated with COPD whereas serum CRP and plasma fibrinogen levels were not. Furthermore, a score for COPD diagnosis with excellent discriminant values was identified, with the best scale for the diagnosis of COPD being obtained using SP-D levels, socio-demographic characteristics, smoking status and respiratory symptoms significantly associated with COPD.

The particular selection of patients and controls allowed us to support our hypothesis that SP-D is able to differentiate COPD patients among individuals consulting for respiratory diseases or symptoms, including those with asthma. COPD and asthma patients, healthy controls and outpatients consulting for non-respiratory diseases were recruited. Regarding the study limitations, the effect of ICS on SP-D levels was assessed by regrouping patients receiving
Table IV. Coordinates of the receiver-operating characteristic curve for all COPD patients and controls.

\begin{tabular}{|c|c|c|}
\hline COPD positive if $\geq$ & Sensitivity & 1-specificity \\
\hline 5.0000 & 1.000 & 1.000 \\
\hline 6.5000 & 0.986 & 0.913 \\
\hline 8.0000 & 0.986 & 0.772 \\
\hline 9.5000 & 0.958 & 0.671 \\
\hline 10.5000 & 0.931 & 0.470 \\
\hline 11.5000 & 0.889 & 0.342 \\
\hline 12.5000 & 0.889 & 0.336 \\
\hline 13.5000 & 0.861 & 0.235 \\
\hline 15.5000 & 0.764 & 0.107 \\
\hline 17.5000 & 0.694 & 0.087 \\
\hline 19.0000 & 0.694 & 0.074 \\
\hline 20.5000 & 0.681 & 0.060 \\
\hline 22.5000 & 0.667 & 0.047 \\
\hline 24.5000 & 0.639 & 0.047 \\
\hline 26.5000 & 0.597 & 0.040 \\
\hline 36.5000 & 0.569 & 0.040 \\
\hline 45.5000 & 0.556 & 0.040 \\
\hline 47.0000 & 0.542 & 0.034 \\
\hline 48.5000 & 0.542 & 0.027 \\
\hline 49.5000 & 0.486 & 0.020 \\
\hline 51.5000 & 0.431 & 0.013 \\
\hline 56.5000 & 0.403 & 0.013 \\
\hline 62.0000 & 0.306 & 0.013 \\
\hline 67.5000 & 0.278 & 0.013 \\
\hline 71.5000 & 0.264 & 0.013 \\
\hline 73.0000 & 0.250 & 0.013 \\
\hline 74.5000 & 0.236 & 0.013 \\
\hline 75.5000 & 0.194 & 0.000 \\
\hline 77.0000 & 0.181 & 0.000 \\
\hline 78.5000 & 0.153 & 0.000 \\
\hline 80.0000 & 0.125 & 0.000 \\
\hline 81.5000 & 0.111 & 0.000 \\
\hline 83.5000 & 0.097 & 0.000 \\
\hline 85.5000 & 0.083 & 0.000 \\
\hline 87.5000 & 0.069 & 0.000 \\
\hline 101.5000 & 0.056 & 0.000 \\
\hline 119.5000 & 0.042 & 0.000 \\
\hline 127.0000 & 0.028 & 0.000 \\
\hline 130.0000 & 0.000 & 0.000 \\
\hline
\end{tabular}

COPD, chronic obstructive pulmonary disease. The value in bold is the optimum cut-off point.

$\beta 2$-agonists (or anticholinergics) combined or not with ICS due to sample size and to the percentage of COPD patients in the severe and very severe stages of the disease, thus requiring treatment with corticosteroids. SP-D levels were lower in the group of COPD patients that were receiving treatment compared with those that were not receiving treatment, but the association did not reach the level of significance reached 


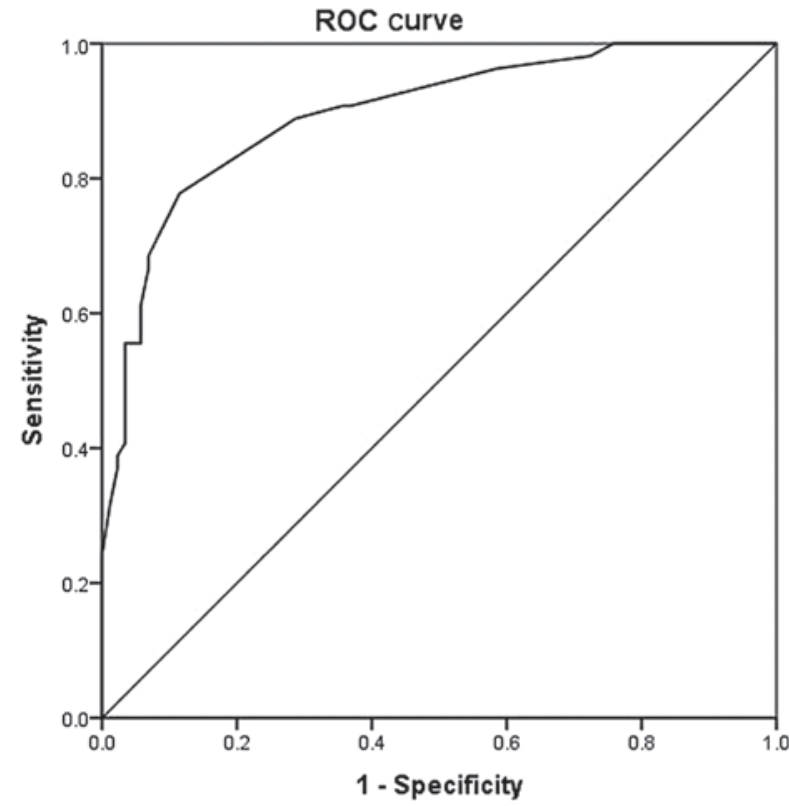

Figure 2. Receiver-operating characteristic (ROC) curve for ever-smoker patients with chronic obstructive pulmonary disease and controls. Diagonal segments are produced by ties.

by previous studies $(12,22,23)$. The present study may have a selection bias $(75.8 \%$ of COPD patients were ever smokers vs. $62.2 \%$ of controls) but this did not affect the analytical results. Recall bias might be possible because information on previous smoking history was based on self-reports. The information bias introduced by underreporting is probable, as smoking behaviors are sensitive issues, particularly among patients that do not want to stop smoking. However, as the patients knew that the study outcome may be beneficial in their medical follow up, it was assumed that the information bias was minimized.

The present study found that SP-D levels were significantly and positively associated with COPD as compared with controls, a result in line with previous studies $(11,12,24)$. To the best of our knowledge, the present study is the first to confirm such a result through multivariate analyses. The association between SP-D and COPD has previously been investigated by bivariate analyses $(11,24-26)$. Ou et al did not find an association between SP-D and COPD in bivariate and multivariate analyses (27) and Ilumets et al adjusted for a single confounding factor, which is age (21). Others have performed multivariate analyses concerning associations of SP-D, but considering COPD exacerbation rather than diagnosis $(12,22,27)$. Since smoking is known to highly affect SP-D levels, the present study considered ever smokers only, and it was found that SP-D remained significantly and positively associated with COPD, a result concordant with previous literature $(11,12,28)$. No association of CRP or fibrinogen with COPD was found in the present study. Ju et al previously reported no association between CRP and stable COPD (24), while other studies have reported higher CRP or fibrinogen levels in stable COPD patients compared with controls (29-31). However, the use of CRP and fibrinogen as biomarkers for COPD is limited by their low specificity and low predictive value for COPD (16) and any inflammatory
Table V. Coordinates of the receiver-operating characteristic curve for ever-smoker COPD patients and controls.

\begin{tabular}{rcc}
\hline COPD positive if $\geq$ & Sensitivity & 1 -specificity \\
\hline 6.0000 & 1.000 & 1.000 \\
8.5000 & 1.000 & 0.759 \\
10.5000 & 0.981 & 0.724 \\
12.0000 & 0.963 & 0.586 \\
13.5000 & 0.907 & 0.368 \\
15.0000 & 0.907 & 0.356 \\
16.5000 & 0.889 & 0.287 \\
$\mathbf{1 8 . 5 0 0 0}$ & $\mathbf{0 . 7 7 8}$ & $\mathbf{0 . 1 1 5}$ \\
21.5000 & 0.685 & 0.069 \\
24.5000 & 0.667 & 0.069 \\
27.5000 & 0.611 & 0.057 \\
36.0000 & 0.574 & 0.057 \\
44.5000 & 0.556 & 0.057 \\
46.5000 & 0.556 & 0.034 \\
48.0000 & 0.537 & 0.034 \\
49.5000 & 0.519 & 0.034 \\
51.0000 & 0.500 & 0.034 \\
52.5000 & 0.481 & 0.034 \\
54.5000 & 0.463 & 0.034 \\
57.5000 & 0.426 & 0.034 \\
60.0000 & 0.407 & 0.034 \\
62.5000 & 0.389 & 0.023 \\
64.5000 & 0.370 & 0.023 \\
66.0000 & 0.315 & 0.011 \\
69.0000 & 0.241 & 0.000 \\
72.5000 & 0.204 & 0.000 \\
77.0000 & 0.093 & \\
95.0000 & 0.056 & \\
113.0000 & 0.037 & \\
117.0000 & 0.000 & \\
\hline
\end{tabular}

COPD, chronic obstructive pulmonary disease. The value in bold is the optimum cut-off point.

or infectious condition, even if not related to lung inflammation, can modify CRP levels (32). In the present study, SP-D levels were also significantly elevated in COPD patients as compared with asthma patients, a result in agreement with a previous study by Mutti et al (26). The present study observed no significant differences in SP-D levels between asthma patients and controls. To the best of our knowledge, there is no clear evidence regarding the association between SP-D and asthma. One study has reported no association of SP-D levels with asthma (26), while some small clinical/case studies have indicated that raised serum SP-D levels are associated with allergy $(29,33)$. Finally, SP-D appears to be a clinical biomarker for COPD, able to differentiate COPD patients among individuals consulting for respiratory diseases or symptoms including those with asthma, while serum CRP and plasma fibrinogen levels are not able to do. 
The present study did not find an association between SP-D and FEV1\% predicted or FEV1/FVC in COPD patients. Previous studies have not reported clear evidence of an association between biomarker levels and lung function parameters. Some studies have reported similar results, whether in bivariate analyses $(12,34,35)$ or in multivariate analysis (36). However, others have described significant negative correlations between SP-D levels and lung functions in bivariate analyses with a borderline P-value for the association between SP-D and FEV1\% predicted (37) or a weak but significant association with FEV1/FVC in only 20 COPD smokers (11). Others have found an association with FEV1 through multivariate analyses in only 23 patients with advanced COPD (38), in severe COPD patients (24) or in smokers only (39). Regarding CRP and fibrinogen, the results of the present study are concordant with previous studies that found no association with FEV1\% in COPD patients $(38,40)$. By contrast, others have found an inverse correlation between CRP or fibrinogen and lung function test results $(7,36)$. Differences between the studies may be explained in part by differences in the sample size, inclusion or exclusion of non-smoker COPD patients and the severity of the disease. In the present study, $81 \%$ of the COPD patients were classified in groups $\mathrm{A}$ and $\mathrm{B}$ and $19 \%$ in groups $\mathrm{C}$ and $\mathrm{D}$, and smoker, ex-smoker and non-smoker COPD patients were included.

To the best of our knowledge, the present study is the first to suggest a score for COPD diagnosis with excellent discriminant values and validity results (area under the curve, sensitivity, specificity, positive predictive value and negative predictive value). There are validated diagnosis scales that could be used in primary care settings without blood measurements such as DS-COPD (diagnosis score for COPD patients) (41). A previous study has created ROC curves based on 44 stable COPD patients to evaluate the diagnostic accuracy of SP-D, and found a total area under the curve of 0.734 (21). In that study, in order to obtain more accurate results, ROC curves were generated for two scores calculated from the variables identified to be associated with COPD in the logistic regression models for all COPD patients and controls and for ever-smoker COPD patients and controls, respectively. In addition to SP-D, the formula for calculating the score included socio-demographic characteristics and respiratory symptoms associated with COPD. The areas under the curves were 0.890 and 0.894 for all subjects and ever smokers, respectively, which may be considered as an improvement compared with the results reported in previous literature (21).

In conclusion, SP-D appears to be able to differentiate COPD patients from patients consulting for other respiratory symptoms or diseases. Used along with socio-demographic characteristics and respiratory symptoms associated with COPD, SP-D is able to discriminate COPD patients from controls, particularly among smokers.

\section{Acknowledgements}

The study was funded by the National Council for Scientific Research (CNRS)-Lebanon, the Doctoral School for Sciences and Technology (EDST)-Lebanon, Novartis-Lebanon and GlaxoSmithKline (GSK)-Lebanon. The European Respiratory Society (ERS) provided a short-term fellowship to the University of Southern Denmark to initiate the study. The authors are grateful to Dr Hasnaa Bou Haroun-Tayoun (Laboratory of Immunology, Faculty of Public Health, Lebanese University) for valuable discussions.

The authors also thank the funding institutions in Lebanon, namely the CNRS, EDST, Novartis and GSK, as well as the European Respiratory Society (ERS) for their unrestricted educational grants.

\section{References}

1. Global Initiative for Chronic Obstructive Lung Disease (GOLD): Global Strategy for the Diagnosis, Management and Prevention of COPD, 2013. http://www.goldcopd.org/. Accessed October 17, 2013.

2. Vestbo J, Anderson W, Coxson HO, Crim C, Dawber F, Edwards L, Hagan G, Knobil K, Lomase DA, MacNee W, et al: Evaluation of COPD longitudinally to identify predictive surrogate end-points (ECLIPSE). Eur Respir J 31: 869-873, 2008.

3. Celli BR and MacNee W; ATS/ERS Task Force: Standards for the diagnosis and treatment of patients with COPD: A summary of the ATS/ERS position paper. Eur Respir J 23: 932-946, 2004.

4. Faner R, Tal-Singer R, Riley JH, Celli B, Vestbo J, Macnee W, Bakke P, Calverley PM, Coxson H, Crim C, et al: Lessons from ECLIPSE: A review of COPD biomarkers. Thorax 69: 666-672, 2014.

5. Engström G, Segelstorm N, Ekberg-Aronsson M, Nilsson PM, Lindgärde F and Löfdahl CG: Plasma markers of inflammation and incidence of hospitalisations for COPD: Results from a population-based cohort study. Thorax 64: 211-215, 2009.

6. Rhim T, Choi YS, Nam BY, Uh ST, Park JS, Kim YH, Paik YK and Park CS: Plasma protein profiles in early asthmatic responses to inhalation allergen challenge. Allergy 64: 47-54, 2009.

7. Dahl M, Tyboerg-Hansen A, Vestbo J, Lang $P$ and Nordestgaad BG: Elevated plasma fibrinogen associated with reduced pulmonary function and increased risk of chronic obstructive pulmonary disease. Am J Respir Crit Care Med 164: 1008-1011, 2001.

8. Thomsen M, Ingebrigtsen TS, Marott JL, Dahl M, Lange P, Vestbo $\mathrm{J}$ and Nordestgaard BG: Inflammatory biomarkers and exacerbations in chronic obstructive pulmonary disease. JAMA 309: 2353-2361, 2013.

9. Man SF, Connett JE, Anthonisen NR, Wise RA, Tashkin DP and Sin DD: C-reactive protein and mortality in mild to moderate chronic obstructive pulmonary disease. Thorax 61: 849-853, 2006.

10. Duvoix A, Dickens J, Haq I, Mannino D, Miller B, Tal-Singer R and Lomas DA: Blood fibrinogen as a biomarker of chronic obstructive pulmonary disease. Thorax 68: 670-676, 2013.

11. Winkler C, Atochina-Vasserman EN, Holz O, Beers MF, Erpenbeck VJ, Krug N, Roepcke S, Lauer G, Elmlinger M and Hohlfeld JM: Comprehensive characterisation of pulmonary and serum surfactant protein D in COPD. Respir Res 12: 29, 2011.

12. Lomas DA, Silverman EK, Edwards LD, Locantore NW, Miller BE, Horstman DH and Tal-Singer R; Evaluation of COPD Longitudinally to Identify Predictive Surrogate Endpoints study investigators: Serum surfactant protein D is steroid sensitive and associated with exacerbations of COPD. Eur Respir J 34: 95-102, 2009.

13. Madsen J, Kliem A, Tornoe I, Skjodt K, Koch C and Holmskov U: Localization of lung surfactant protein $\mathrm{D}$ on mucosal surfaces in human tissues. J Immunol 164: 5866-5870, 2000.

14. Crouch EC: Surfactant protein-D and pulmonary host defense. Respir Res 1: 93-108, 2000.

15. Hoegh SV, Sorensen GL, Tornoe I, Lottenburger T, Ytting H, Nielsen HJ, Junker P and Holmskov U: Long-term stability and circadian variation in circulating levels of surfactant protein D. Immunobiology 215: 314-320, 2010.

16. Antoniu SA: Effects of inhaled therapy on biomarkers of systemic inflammation in stable chronic obstructive pulmonary disease. Biomarkers 15: 97-103, 2010.

17. Global Initiative for asthma (GINA): Global Strategy for Asthma Management and Prevention, 2012. http://www.ginasthma.org/. Accessed November 18, 2013.

18. Ferris BG: Epidemiology Standardization Project (American Thoracic Society). Am Rev Respir Dis 118: 1-120, 1978. 
19. Stenton C: The MRC breathlessness scale. Occup Med (Lond) 58: 226-227, 2008.

20. Leth-Larsen R, Nordenbaek C, Tornoe I, Moeller V, Schlosser A, Koch C, Teisner B, Junker P and Holmskov U: Surfactant protein $\mathrm{D}$ (SP-D) serum levels in patients with community-acquired pneumonia. Clinical Immunol 108: 29-37, 2003.

21. Ilumets H, Mazur W, Toljamo T, Louhelainen N, Nieminen $P$, Kobayashi H, Ishikawa N and Kinnula VL: Ageing and smoking contribute to plasma surfactant proteins and protease imbalance with correlations to airway obstruction. BMC Pulm Med 11: 19, 2011.

22. Foreman MG, Kong X, DeMeo DL, Pillai SG, Hersh CP, Bakke P, Gulsvik A, Lomas DA, Litonjua AA, Shapiro SD, et al: Polymorphisms in surfactant protein-D are associated with chronic obstructive pulmonary disease. Am J Respir Cell Mol Biol 44: 316-322, 2011.

23. Sin DD, Man SF, Marciniuk DD, Ford G, FitzGerald M, Wong E, York E, Mainra RR, Ramesh W, Melenka LS, et al: The effects of fluticasone with or without salmeterol on systemic biomarkers of inflammation in chronic obstructive pulmonary disease. Am J Respir Crit Care Med 177: 1207-1214, 2008.

24. Ju CR, Liu W and Chen RC: Serum surfactant protein D: Biomarker of chronic obstructive pulmonary disease. Dis Markers 32: 281-287, 2012.

25. Shakoori TA, Sin DD, Ghafoor F, Bashir S and Bokhari SN Serum surfactant protein D during acute exacerbations of chronic obstructive pulmonary disease. Dis Markers 27: 287-294, 2009.

26. Mutti A, Corradi M, Goldoni M, Vettori MV, Bernard A and Apostoli P: Exhaled metallic elements and serum pneumoproteins in asymptomatic smokers and patients with COPD or asthma. Chest 129: 1288-1297, 2006.

27. Ou CY, Chen CZ, Hsiue TR, Lin SH and Wang JY: Genetic variants of pulmonary SP-D predict disease outcome of COPD in a Chinese population. Respirology 20: 296-303, 2015.

28. Sorensen GL, Husby S and Holmskov U: Surfactant protein A and surfactant protein $\mathrm{D}$ variation in pulmonary disease. Immunobiology 212: 381-416, 2007.

29. Inase N, Ohtani Y, Sumi Y, Umino T, Usui Y, Miyake S and Yoshizawa Y: A clinical study of hypersensitivity pneumonitis presumably caused by feather duvets. Ann Allergy Asthma Immunol 96: 98-104, 2006.

30. de Torres JP, Cordoba-Lanus E, López-Aguilar C, Muros de Fuentes M, Montejo de Garcini A, Aguirre-Jaime A, Celli BR and Casanova $\mathrm{C}$ : C-reactive protein levels and clinically important predictive outcomes in stable COPD patients. Eur Respir J 27: 902-907, 2006
31. Eickhoff P, Valipour A, Kiss D, Schreder M, Cekici L, Geyer K, Kohansal R and Burghuber OC: Determinants of systemic vascular function in patients with stable chronic obstructive pulmonary disease. Am J Respir Crit Care Med 178: 1211-1218, 2008.

32. Kushner I, Rzewnicki D and Samols D: What does minor elevation of C-reactive protein signify? Am J Med 119: 166. e17-e28, 2006.

33. Tsushima K, Fujimoto K, Yoshikawa S, Kawakami S, Koizumi T and Kubo K: Hypersensitivity pneumonitis due to Bunashimeji mushrooms in the mushroom industry. Int Arch Allergy Immunol 137: 241-248, 2005.

34. Um SJ, Lam S, Coxson H, Man SF and Sin DD: Budesonide/ formoterol enhances the expression of pro Surfactant Protein-B in lungs of COPD patients. PloS One 8: e83881, 2013.

35. Liu W, Ju CR, Chen RC and Liu ZG: Role of serum and induced sputum surfactant protein $\mathrm{D}$ in predicting the response to treatment in chronic obstructive pulmonary disease. Exp Ther Med 8: 1313-1317, 2014

36. Engström G, Lindberg C, Gerhardsson de Verdier M, Nihlén U, Anderson M, Svartengren M and Forsman-Semb K: Blood biomarkers and measures of pulmonary function - a study from the Swedish twin registry. Respir Med 106: 1250-1257, 2012.

37. Ozyurek BA, Ulasli SS, Bozbas SS, Bayraktar N and Akcay S: Value of serum and induced sputum surfactant protein-D in chronic obstructive pulmonary disease. Multidiscip Respir Med 8: 36, 2013

38. Sin DD, Leung R, Gan WQ and Man SP: Circulating surfactant protein $\mathrm{D}$ as a potential lung-specific biomarker of health outcomes in COPD: A pilot study. BMC Pulm Med 7: $13,2007$.

39. Johansson SL, Tan Q, Holst R, Christiansen L, Hansen NC, Hojland AT, Wulf-Johansson H, Schlosser A, Titlestad IL, Vestbo J, et al: Surfactant protein D is a candidate biomarker for subclinical tobacco smoke-induced lung damage. Am J Physiol Lung Cell Mol Physiol 306: L887-L895, 2014.

40. Dickens JA, Miller BE, Edwards LD, Silverman EK, Lomas DA and Tal-Singer R; Evaluation of COPD Longitudinally to Identify Surrogate Endpoints (ECLIPSE) study investigators: COPD association and repeatability of blood biomarkers in the ECLIPSE cohort. Respir Res 12: 146, 2011.

41. Salameh P, Khayat G and Waked M: Could symptoms and risk factors diagnose COPD? Development of a Diagnosis Score for COPD. Clin Epidemiol 4: 247-255, 2012. 